\title{
NOTES ON COHERENT POLARIZATION X-RAY RADIATION BY RELATIVISTIC ELECTRONS IN A CRYSTAL
}

\author{
V. L. Morokhovskii* \\ National Science Center "Kharkiv Institute of Physics and Technology", 61108, Kharkiv, Ukraine
}

(Received August 20, 2018)

\begin{abstract}
This paper deals with restrictions on the use of so-called kinematical theory of coherent polarization X-ray radiation
\end{abstract} by relativistic electrons in a single crystal target.

PACS: 03.65.Pm, 03.65.Ge, 61.80.Mk

\section{INTRODUCTION}

It is common knowledge that the collision of relativistic charged particles with atoms and nuclei results in the emission of electromagnetic waves. The radiation consists of two components. The first one is the radiation emitted directly by the charged particle in the Coulomb field of the nucleus and its surrounding atomic electrons. This part is well known as the Bethe-Heitler bremsstrahlung. The second contribution is caused by elastic scattering of the virtual photons associated with the relativistic charged particle at the atomic electrons and nuclei. It has been called polarization bremsstrahlung or simply polarization radiation. Comparing both parts it is worth to remark that while the part of bremsstrahlung, described by Bethe-Heitler expressions, is emitted mainly into the angle of order of $1 / \gamma$, with $\gamma$ being the relativistic Lorentz factor in the direction of the particle velocity. Polarization radiation appears, although with varying intensity, at any direction $[1,2,3]$.

The radiation pattern changes drastically if particle interacts not just with atom or an amorphous medium, but with the constituents of a crystal.

In this case the charged particle passes with a nearly constant velocity through the spatial periodically distributed atoms. Due to the electromagnetic interaction, the charged particle and the atomic electrons become sources of electromagnetic waves. And due to the space periodicity of the crystal media and the time periodicity of the particle-atomic collisions, the constructive interference of these waves exists.

The constructive interference results in the appearance of coherent $\gamma$-radiation in the case of radiation emitted by the charged particle, i.e. coherent bremsstrahlung (CB) and coherent X-ray polarization radiation $(\mathrm{CPR})^{1}$ in the case of emission by atomic electrons, respectively. $\mathrm{CB}$ is emitted mainly into the angle of order of $1 / \gamma$ in the direction of the particle velocity. CPR emitted mainly into the angle of order of $1 / \gamma$ in different directions near the Bragg angles, which are corresponded to the elastic scattering of the virtual photons associated with the relativistic charged particle at the different crystal planes with low Miller indexes. Such properties of $\mathrm{CB}$ and CPR give opportunity to separate these two types of coherent radiations by the choice the angle of photon observation $\theta_{k} \gg 1 / \gamma$ and angle between electron momentum $\vec{p}_{i}$ and the reflected crystal plane in the region $\theta_{k} / 2-2 / \gamma \leq \phi \leq \theta_{k} / 2+2 / \gamma$.

In 1987 the author proposed "The way (method) of generation of the monochromatic directed X-ray radiation", based on the properties of the CPR phenomenon, and got the "Invention's Certificate" [4]. The properties of CPR have been predicted in [6] in the framework of the theoretical model, which is called "Kinematical theory" and has been at first confirmed by experiments at Kharkiv Linear Electron Accelerators. This work was the physical ground of the invention [4]. Thus in $[4,6]$ it was proposed the smoothly-turnable highly-monochromatic and highly-polarized X-ray source with negligibly small contribution of the continuous background, and adequate theoretical description of the CPR phenomenon was made.

The CPR phenomenon then was investigated experimentally and theoretically in detail by international collaboration in Darmstadt (Germany) at the Superconductor Darmstadt Linear Electron Accelerator S-DALINAC $[7,8,9,10,11,12,13,15]$. In [12] it was shown, that all properties of CPR, which were measured in absolute units in Kharkiv, Darmstadt, Tomsk, Tokyo and the USA, were calculated and found nearly the perfect agreement between the

\footnotetext{
${ }^{*}$ Corresponding author E-mail address: victor@kipt.kharkov.ua

${ }^{1}$ The historical circumstances put to some different names of this phenomenon. In the literature are used the names: "Parametric X-radiation (PXR)"; or "Parametric X-radiation type B (PXR(B))", where abbreviation (B) means the word "Bremsstrahlung".
} 
theory of perturbation and all referred experimental results.

In particular, in [9] it was compared the intensity of channeling radiation (CR) and intensity of CPR from the same diamond target and at the same electron energy $\left(3.0 \mathrm{MeV} \leq E_{e} \leq 9.0 \mathrm{MeV}\right)$ and it was shown, that the intensity of CPR is three order of magnitude weaker than $\mathrm{CR}$ under the same conditions.

In the publication [22] it was considered theoretically the case of CPR emitted by channeling electrons with energies of several $\mathrm{MeV}$, which during the radiation process make the transition between two bound states. Authors of this publication advocated: "we have an X-ray emission as a result of diffraction of virtual CR", and they called this radiation process as "diffracted channeling radiation (DCR)". Furthermore the authors of [22] made the statement: "It is shown that, in comparison with PXR, the spectral density of DCR is very large and the width of the angular distribution is very narrow. The peak intensity of DCR is about 10 times larger than that of PXR".

The geometry of such radiation process is shown in Fig.1.

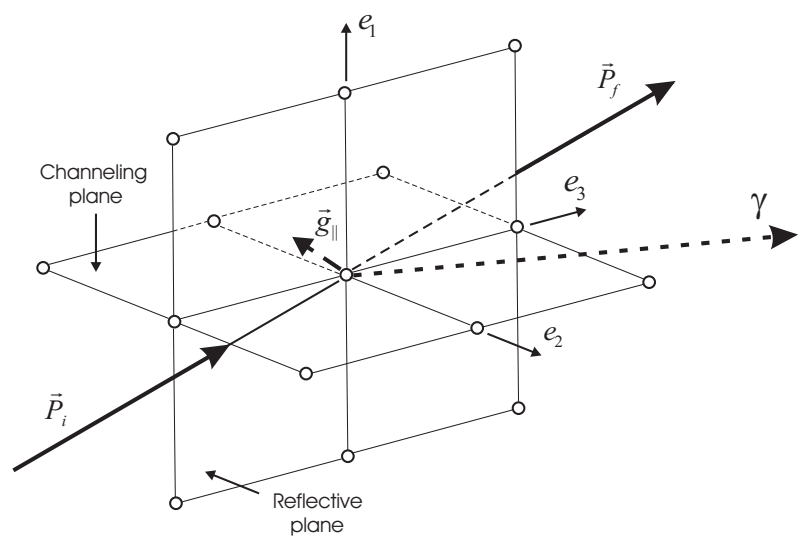

Fig.1. Geometry of the experiment. The relativistic electron with momentum $\vec{p}_{i}$, which is parallel to the crystal plane $\left(\vec{e}_{2}, \vec{e}_{3}\right)$, penetrates into the crystal and channeling in the plane $\left(\vec{e}_{2}, \vec{e}_{3}\right)$. The virtual photon emitted by the channeling electron and reflected by the plane $\left(\vec{e}_{1}, \vec{e}_{3}\right)$, which is perpendicular to the channeling plane and creates the angle $\angle\left(\vec{p}_{i}, \vec{e}_{3}\right) \approx \theta_{B}$ $\left(\theta_{B}\right.$ is the Bragg angle), and the real photon $\gamma$ emitted under the near the mirror angle. Relativistic electron leaves the crystal with momentum $\vec{p}_{f}$. The crystal got the recoil momentum, which is equal to the reciprocal lattice vector $\vec{g}_{\|}$

Intuition suggests that according to the physical pattern of the CPR phenomenon, which is described above, that the ideal dynamic of the incident relativistic electron, which gives the most intensive CPR, is the uniform straight line motion. It is no doubt that the character of charged particle dynamics has an influence on the the coherent length in the coherent radiation process. In [8] it's shown, that the deviations of the relativistic electron motion from the uniform straight line motion cause the value of the CPR intensity to decrease. The phenomenon of channeling corresponds to the particulary dynamic of relativistic electron in crystal, when its motion perpendicular to the channeling plane, which is characterized by creation bound states in the averaged plane potential with discrete spectrum of the transverse energies. Thus the statement made in [22] is not in agreement with the habitual physical pattern of the CPR.

The conditions of experiment [9] were very much close to the conditions, which are necessary for observation so-called DCR, but it was not discovered.

Authors of [23] have performed the numerical calculations of the spectral-angular distributions of the DCR and at the end of their article they have formulated the following question: "Why in spite of sizable amplitude of the DCR, it was not discovered up till now?"

Let us make effort to answer this question.

\section{CB+CPR IN THE FRAMEWORK OF THE KINEMATICAL THEORY}

Both differential cross sections of ordinary coherent bremsstrahlung (CB) and coherent polarization radiation (CPR), obtained in the framework of theory of perturbation and used in $[4,5,15,19]$, contain the product of $\delta$-functions: $\delta\left(\vec{p}_{i}-\vec{p}_{f}-\vec{k}-\vec{g}\right) \delta\left(E_{i}-E_{f}-\omega\right)$, which are the consequence of the momentum-energy conservation laws (here the system of units $\hbar=c=1$ is used). It means that: $\vec{p}_{i}$ and $E_{i}$ are momentum and energy of relativistic electron in initial state; $\vec{p}_{f}$ and $E_{f}$ are momentum and energy of the relativistic electron in the final state; $\vec{k}$ and $\omega$ are momentum and energy of the radiated photon; $\vec{q} \equiv \vec{p}_{i}-\vec{p}_{f}-\vec{k}=\vec{g}$ is the recoil momentum received by crystal target; $\vec{g}$ is the reciprocal lattice vector. The kinematic of such processes is shown in Fig.2.
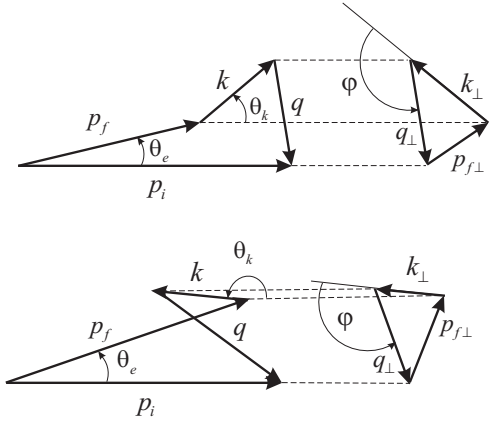

Fig.2. Kinematical sketches of the radiation process of relativistic charged particle interacting with target: $a$ - radiation into the forward half-sphere; $b$ - radiation into the backward half-sphere

For the calculation of the spectral-angular distribution of the coherent $\mathrm{x}$-ray radiation we assume, that the relativistic particle that causes the radiation is not detected. After integration of differential cross section (see section 2 of [29]) over the final states of relativistic electron we obtain the total coherent spectral-angular cross section, normalized to the vol- 
ume of a crystal cell, which looks like:

$$
\begin{gathered}
\left(\frac{d^{3} \sigma}{d \omega d \Omega}\right)_{c o h}=\frac{8 \pi \omega}{V\left(1-\vec{v} \vec{n}_{k}\right)} \sum_{\vec{g}, \lambda}\left|M_{C R}\right|^{2} \times \\
\times S^{2}(\vec{g}) e^{-g^{2} \overline{u^{2}}(T)} \delta\left(\omega-\frac{\vec{g} \vec{v}}{1-\vec{v} \vec{n}_{k}}\right),
\end{gathered}
$$

where $\vec{n}_{k}=\vec{k} / k$, and the sum includes all reciprocal lattice vectors $\vec{g}$ of the crystal and the photon polarization directions $\vec{\epsilon}_{k \lambda}$. So as the charged particle is an electron, i.e. its charge $e_{0}=-|e|$ and its mass $m_{0}=m$, the square of the matrix element module in Eq.(1) is of the form:

$$
\begin{gathered}
\left|M_{C R}\right|^{2}=\left|M^{B S}+M^{P R}\right|^{2}=\frac{e^{6}}{\omega^{2} m^{2}} \times \\
\times\left|\vec{\epsilon}_{k \lambda}\left\{\frac{Z-F(\vec{g})}{\gamma g^{2}} \frac{\vec{g}}{1-\vec{v} \vec{n}_{k}}+F(\vec{g}) \frac{\omega \vec{v}-\vec{g}}{(\vec{k}+\vec{g})^{2}-k^{2}}\right\}\right|^{2} .
\end{gathered}
$$

The first part of the right hand side of Eq.(2) corresponds to the coherent bremsstrahlung of the charged particle $(C B)$ and the second part corresponds to the coherent polarization radiation $(C P R)$ emitted by crystal electrons. Both parts are proportional to $\mathrm{m}^{-2}$, which is to say that both types of radiation have the same bremsstrahlung nature [12, 27, 29].

Due to the condition $\vec{q}=\vec{g}$ for the momentum transferred to the crystal, the X-radiation becomes almost monochromatic. This mechanism has been described in detail for coherent bremsstrahlung in $[24,26,25]$. The second part, i.e. coherent polarization radiation in the sense of the coherent component of the bremsstrahlung produced by atomic electrons, exactly represents CPR (and CPR identical with socalled $\operatorname{PXR}(\mathrm{B})[6,7])$.

\subsection{Influence of the Dielectric Properties of the Crystal}

Matrix elements 2 were calculated neglecting the influence of the dielectric properties of the crystal, i.e. the radiation photons were treated as traveling through vacuum. However, the influence of the crystal can be taken into account by changing the dispersion relation for the radiated photon to $k^{2}=\varepsilon \omega^{2}$, where $\varepsilon=1+\chi$ is the dielectric constant with $\chi$ being the electric susceptibility of the crystal. Thus, using this dispersion relation in Eq.2, we obtain the expression of the square of the matrix element module:

$$
\begin{gathered}
\left|M_{C R}\right|^{2}=\frac{e^{6}}{\varepsilon \omega^{2} m^{2}} \mid \vec{\epsilon}_{k \lambda}\left\{\frac{Z-F(\vec{g})}{\gamma g^{2}} \frac{\vec{g}}{1-\vec{v} \vec{n}_{k}}+\right. \\
\left.+F(\vec{g}) \frac{\sqrt{\varepsilon} \omega \vec{v}-\vec{g}}{\omega^{2}\left(1 /(\beta \gamma)^{2}+1-\varepsilon\right)+\left(\sqrt{\varepsilon} \omega\left(\vec{n}_{k}\right)_{\perp \vec{v}}+\vec{g}_{\perp \vec{v}}\right)^{2}}\right\}\left.\right|^{2} .
\end{gathered}
$$

Comparing Eqs.(2) and (3) it becomes apparent that the influence of the medium effects the matrix element mainly at high particle energies, i.e. if $1 /(\beta \gamma)^{2} \leq|1-\varepsilon|=|\chi|$. For low energies, i.e. if $1 /(\beta \gamma)^{2} \gg|\chi|$, the influence of the medium can be neglected and it turns out that the maximum of the cross section increases proportional to $\sim \gamma^{2}[6,7]$. In contrast to this case, at high energies the cross section is limited by $\chi$ and the shape of the angular distribution becomes constant $[12,21,27] .^{2}$

\section{3. "EFFECT OF ROW"}

If we discuss Eqs.(1) and (2), it can be stated that both CBS and CPR contribute to coherent Xradiation at the same photon energy $\omega-\frac{\vec{g} \vec{v}}{1-\vec{v} \vec{n}_{k}}$, but they are emitted with different angular distributions.

On the basis of laws of momentum-energy conservation one can determine the area of recoil momenta $\vec{q}$, which are allowed by kinematics (see Fig.2) and represent it (so-called "Pancake") inside the momenta space together with the reciprocal lattice of the crystal target. In Fig.3 is shown such region of the momentum space, which confirms with geometry of experiment [9] shown in Fig.1 (the channeling plane is the plane (001) of the diamond crystal, and the main reflected plane is $(2 \overline{2} 0))$. One can see, that in this case laws of momentum-energy conservation allow for the contribution into the radiation process the row of crystal planes, which correspond to the reciprocal lattice vectors $\ldots, \vec{g}_{2 \overline{2} 4}, \vec{g}_{2 \overline{2} 0}, \vec{g}_{2 \overline{2} \overline{4}}, \ldots$

In the case of $\mathrm{CB}[24,26,25]$ all these reciprocal lattice vectors contribute to the intensity emitted forward into the angle cone with an opening angle of $\approx 1 / \gamma$. For CB all angular cones of partial radiations, which are caused by contributions from the series of the reciprocal lattice vectors, are coaxial. Therefore in the case $\mathrm{CB}$ we have the effective summatiom of these partial radiations. This is the sense of the term of "effect of row".

On the other hand, CPR is emitted mainly into the cone of similar angular size but which is aligned to the direction of $\omega \vec{v}-\vec{g}$. For CPR all angular cones of partial radiations, which are caused by contributions from the series of the reciprocal lattice vectors, have different directions and therefore the radiation

\footnotetext{
${ }^{2}$ Our formula 3 and M.L. Ter-Mikalian's formula (28.160) in his book [14], which describes so-called "resonance radiation", are similar in appearance. However they have vital differences. M.L. Ter-Mikalian has supposed, that the crystal dielectric constant must be $\epsilon>1$. Therefore his formula is in the contradiction with laws of momentum-energy conservation. Because of that M.L. Ter-Mikalian's formula (28.160 of [14]) predicts unreal properties of coherent X-radiation, i.e. spectral-angular distribution. "Resonance radiation" is characterized by the energy threshold, which is absent for CPR, and so on. For the repairing M.L. Ter-Mikalian's formula (28.160) it is necessary to assume $\epsilon \leq 1$, to change the sign near the recoil momentum, and to define more precisely the constant multiplier before this formula. In the several theoretical works on PXR made an exact replica of M.L. Ter-Mikalian's formula (28.160), i.e. used erroneous sign near the recoil momentum received by crystal in the CPR process. Therefore formulae (3) in [16], (19) in [18] and (1.1) in [20] are in the contradiction with laws of momentum-energy conservation and do not agree with experiment [17].
} 
caused by the row of reciprocal lattice vectors overlap weakly.

$\langle 110\rangle$
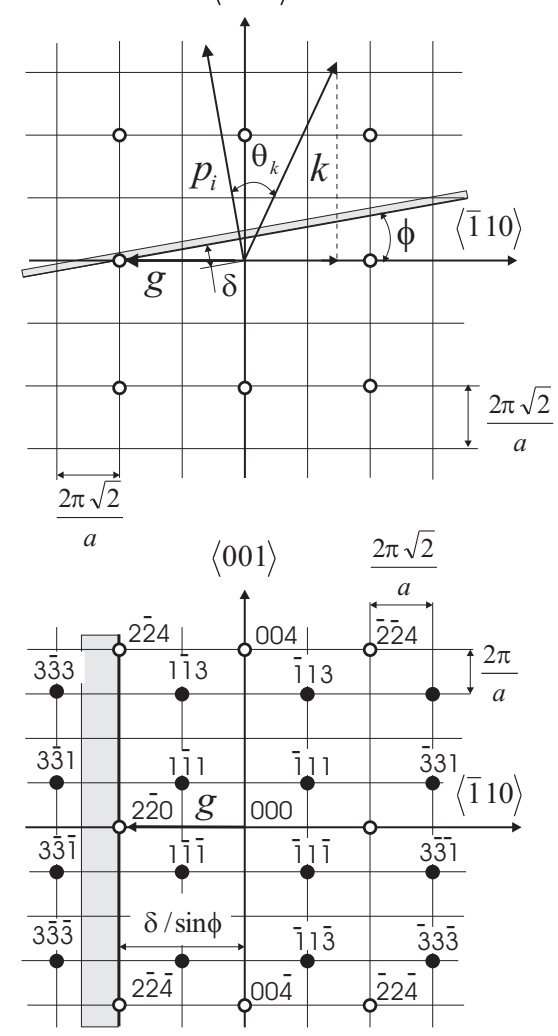

Fig.3. Two projections of crossing the "pancake" of allowed recoil momenta by kinematics and reciprocal lattice of Diamond crystal. Vectors $\vec{p}_{i}$ and $\vec{k}$ are in the plane with Miller indexes (001). "Pancake" is perpendicular to $\vec{p}_{i}$ and placed on the distance $\delta \approx \omega /\left(2 E_{i} E_{f}\right)+\omega\left(1-\cos \theta_{k}\right)$ from the origin of coordinate system. The thickness of "pancake" is $\sim \delta$. The grey vertical strip in the horizontal projection shows the crossing of "pancake" and the main plane of the reciprocal lattice [6]

Thus, for the different reciprocal lattice vectors CPR can be obviously observed at different directions and several individual maxima of coherent radiation appear. For high particle energies these maxima are well separated from each other. In contrast, for low energies, i.e. if $1 / \gamma$ becomes comparable to the angles between two reciprocal lattice vectors, the angular cones corresponding to several reciprocal lattice vectors may partially overlap (Fig.4).

It should be pointed out, furthermore, that in the two extreme cases Eqs.(1) and (2) represent the known, independent representations of $\mathrm{CB}$ and CPR. In the first case, for an observation angle $\theta=\angle\left(\vec{v}, \vec{n}_{k}\right) \approx 1 / \gamma$ the first part of Eq.(2) becomes dominant, and thus Eqs.(1) and (2) describe exactly $\mathrm{CB}$ in the low photon energy approximation analogous to $[24,25,26]$. On the other hand, if $\theta \gg 1 / \gamma$ the contribution of CB becomes small and can be neglected. In this case Eqs.(1) and (2) precisely represent the mathematical description of CPR which has been published before in [6, 7$]$.
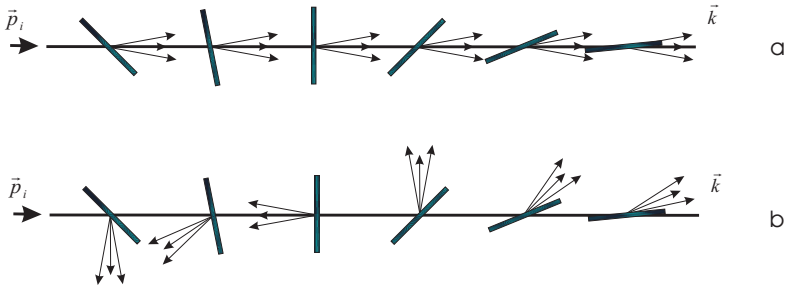

Fig.4. Schematic sketch of radiation contributions from crystal planes with different orientations and with condition $\vec{g}_{i} \vec{v}=\vec{g}_{j} \vec{v}$, where $i, j$ - denote the indexes of planes, which are described by their reciprocal lattice vectors $\vec{g}_{i} ; \vec{v}$ is the charged particle velocity: $a$ - for $C B ; b-$ for $C P R$

If we selected the radiation angle $\theta_{k} \gg 1 / \gamma$, then we eliminated not only CB background, but the contribution of CPR stipulated by the reciprocal lattice vectors, which are not in the plane of radiation. In this case "effect of row" for CPR is absent of fact, and we have "effect of point". So in the case, which is described in Fig.3 we may take into account only dominant contribution of vector $\vec{g}_{2 \overline{2}}$.

\section{CPR GENERATED BY CHANNELING RELATIVISTIC ELECTRONS}

The EM field inside the crystal matter can be written in two-wave approximation in the following form [27]:

$$
\begin{aligned}
\vec{A}_{\omega}(\vec{r})= & \sum_{s=1}^{4} C_{s}\left[\vec{\epsilon}_{1}^{(s)} \exp \left(i \vec{k}_{1} \vec{r}\right)+\right. \\
& \left.+\alpha_{s} \vec{\epsilon}_{2}^{(s)} \exp \left(i \vec{k}_{2} \vec{r}\right)+\text { c.c. }\right],
\end{aligned}
$$

where $\vec{k}_{2}=\vec{k}_{1}+\vec{g}, k=\omega / c^{*}, \alpha_{s}$ denotes the amplitudes of the reflected waves, and $C_{s}$ are constants. $s=(1,2,3,4)$ because of two photon polarizations and two possible refraction factors.

Now let us mean, that the arbitrary reciprocal lattice vector is $\vec{g}=\vec{g}_{\|}+\vec{e}_{1} \cdot g_{h}$, where $\vec{g}_{\|}$is in the channeling plane, and $g_{h}$ is the projection $\vec{g}$ on the unit vector $\vec{e}_{1}$, and suppose that initial electron momentum $\vec{p}^{(i)}$ is parallel to the channeling plane (see Fig.1). The coordinate wave functions of the channeling electron $([28,30,5])$ in the initial and final states may be written in the form of Bloch functions:

$$
\phi^{(m)}(\vec{r})=\sum_{h} A_{h}^{(m)} e^{i\left(\vec{p}_{\|}^{(m)}+\vec{e}_{1} \cdot g_{h}\right) \vec{r}} .
$$

Passing over the constant, matrix element $M_{C R}$ can be written in the form:

$$
M_{C R} \sim \int d^{3} r \phi_{f}^{*}(\vec{r}) \hat{\vec{p}} \cdot \vec{A}_{\omega}^{*}(\vec{r}) \phi_{i}(\vec{r}) .
$$

Substituting $\vec{A}_{\omega}^{*}(\vec{r})$ in the form of (4) and $\phi_{i,(f)}(\vec{r})$ in the form of Bloch functions, we obtain sum of two matrix elements, which origin from two terms with $\vec{k}_{1}$ and $\vec{k}_{2}$ in the sum of (4). These matrix elements differ from each other by $\delta$-functions: $\delta\left(\vec{p}_{\|}^{(i)}-\vec{p}_{\|}^{(f)}-\vec{k}_{1,(2) \|}\right)$. The first matrix element represents the ordinary channeling radiation. The second 
one describes the coherent polarization radiation of the channeling electron.

If we suppose wave functions $\phi_{i,(f)}(\vec{r})$ to be plane waves, we obtain result, which exactly coincides with (3) (see [27]).

Insertion of the EM field component $\vec{A}_{\omega}^{(s)}(\vec{r})$ from Eq.(4) and channeling electron wave functions in the form of (5) into Eq.(6) gives:

$$
\begin{aligned}
& M_{C R}^{(s)} \sim \alpha_{s} \vec{\epsilon}_{2}^{(s)} \sum_{h, h^{\prime}} A_{h}^{(m)}\left(\vec{p}_{\|}^{(i)}-\vec{k}_{1}-\vec{g}_{\|}-\vec{e}_{1} g_{h}\right) A_{h^{\prime}}^{\left(m^{\prime}\right)} \\
& \times \delta\left(\vec{p}_{\|}^{(i)}-\vec{p}_{\|}^{(f)}-\vec{k}_{\|}-\vec{g}_{\|}\right) \delta\left(g_{h}-p_{\perp}^{(f)}-k_{\perp}^{(1)}-g_{h^{\prime}}\right),
\end{aligned}
$$

where $p_{\perp}^{(f)}=\vec{p}_{f} \cdot \vec{e}_{1}$ and $k_{\perp}^{(1)}=\vec{k}_{1} \cdot \vec{e}_{1}$. After integration $\left|M_{C R}^{(s)}\right|^{2}$ over $d^{3} p_{f}$ we obtain the multitude of reflections in different crystallographic planes, which do not contribute into selected direction of photon registration. So effective summation of different reflections is absent and the situation occurs analogous to depicted above in the subsection "EFFECT OF ROW".

\section{CONCLUSIONS}

It is safe to repeat the following quotation from the paper [12]: "So it's shown, that simple and clear so-called kinematical theory $[6,13]$ is enough for exact calculation of all properties of the present type of radiation".

\section{References}

1. M.Ya. Amus'ya, V.M. Buimistrov, B.A.Zon, et al. Polarization bremsstrahlung of particles and atoms. Moskow: "Nauka". 1980, 335p. (in Russian).

2. M.Ya. Amus'ya, M.Yu. Kuchiev, A.V. Korol', and A.V.Solov'ev. Bremsstrahlung of relativistic particles and dynamic polarizability of target atom // Zh. Eksp. Teor. Fiz. 1985, v.88, p.383-389.

3. V.A. Astapenko,

V.M. Buimistrov, Yu.A. Korotkov, L.K. Mikhailov, and L.I. Trakhtenberg. Dynamic bremsstrahlung of relativistic charged particle scattered by atom // Zh. Eksp. Teor. Fiz. 1985, v.88, p.1560-1569.

4. V.L. Morokhovskii, et al. The way (method) of generation of the monochromatic directed $X$-ray radiation: Author's certificate of the invention N1513528, Bulletin USSR Discovery, Inventions, N37, p.51, from October 07, 1989, (priority from April 13 1987).

5. V.L. Morokhovskii. Wave functions of the bound states of relativistic electrons in crystal for electron movement along the crystal plane: Preprint, M: "CNRIatominform". 1987, 6 p. (in Russian).

6. V.L. Morokhovskii. Coherent X-rays of Relativistic Electrons in Crystals: Preprint, Moskow: "CNRIatominform", 1989, 39 p. (in Russian).
7. J. Freudenberger, V. B. Gavrikov, M. Galemann, H. Genz, L. Groening, V. L. Morokhovskii, V. V. Morokhovskii, U. Nethin, A. Richter, J.P. F. Selshop, N. F. Shul'ga. Parametric X-ray Radiation Obseved in Diamond at Law Electron Energies // Physical Review Letters. 1995, v.74, N13, p.2487-2490.

8. J. Freudenberger, H. Genz, V. V. Morokhovskii, A. Richter, V.L. Morokhovskii, U. Nething, R. Zahn, J.P.F. Sellschop. Lineshape, linewidth and spectral density of parametric x-radiation at low electron energy in diamond // Appl. Phys, Lett. 1997, N70(2), p.267-269.

9. J. Freudenberger, H. Genz, L. Groening, P. Hofman-Stascheck, W. Knüpfer, V.L. Morokhovskii, $\quad$ V.V. Morokhovskii, U. Nething, A. Richter, J.P.F. Sellschop. Canneling radiation and parametric $\mathrm{X}$-radiation at electron energies below $10 \mathrm{MeV} / /$ Nuclear Instruments and Methods in Physics Research B. 1996, v.119. p.123-130.

10. V.V. Morokhovskyi, K.H. Shmidt, G. Buschhorn, J. Freudenberger, H. Genz, R. Kotthaus, A. Richter, M. Rzepka, and P.M. Weinmann. Polarization of Parametric X Radiation // Physical Review Letters, 1997, v.79, N22, p.4389-4392.

11. V.V. Morokhovskyi, K.H.Shmidt, G. Buschhorn, J. Freudenberger, H. Genz, R. Kotthaus, A. Richter, M. Rzepka, and P.M. Weinmann. Polarization of parametric X radiation // Nuclear Instruments and Methods in Physics Research B. 1998 , v.145. p.14-18.

12. J. Freudenberger, H. Genz, V.L. Morokhovskii, V. V. Morokhovskii, A. Richter, J.P. F. Selshop. Properties of Coherent X-Radiation Produced by Relativistic Charged Particles Passing through a Crystal: Preprint IKP TUD, Darmstadt, 1997, $77 \mathrm{p}$.

13. V. V. Morokhovskii, J. Freudenberger, H. Genz, V. L. Morokhovskii, A. Richter, J.P. F. Selshop. Theoretical description and experimental determination of the interference between parametric $\mathrm{X}$ radiation and coherent bremsstahlung // Phys. Rev.B. 2000-I, v.61, N1, p.3347-3352.

14. M.L. Ter-Mikhaelyan. High-Energy Electromagnetic Processes in Condensed Media. New York: Wiley-Interscience, 1972, 457p.

15. D. Pugachov, G. Buschhorn, R. Kotthaus, V.L. Morokhovskii, J. They, H. Genz, A. Richter, A. Ushakov. Polarization properties of coherent polarisation radiation of relativistic electrons in a crystal // Physics Letters. 2001, v.A286, p.70-75. 
16. I.D. Feranchuk and A.V. Ivvashin. Theoretical investigation of the parametric X-ray features // J. Phys. Paris, 1985, v.46, p.1981-1986.

17. S. Assano, I. Endo, M. Harada, S. Ishii, T.Kobayashi, T. Nagata, M. Muto, K. Yoshida, H. Nitta. How Intense is Parametric X Radiation? // Phys. Rev. Lett. 1993, v.70, N21, p.3247-3250.

18. H. Nitta. Kinematical theory of parametric X-ray radiation // Physics Letters. 1991, v.A158, p.270274 .

19. H. Nitta. Theory of coherent X-ray radiation by relativistic particles in a single crystal // Phys. Rev.B. 1992-II, v.45, N4, p.7621-7626.

20. H. Nitta. Theoretical notes on parametric X-ray radiation // Nuclear Instruments and Methods in Physics Research B. 1996, v.115. p.401-404.

21. H. Nitta. Dynamical effect of parametric X-ray radiation // Journal of the Physical Society of Japan. 2000, v.69, N10, p.3462-3465.

22. R. Yabuki, H. Nitta, T. Ikeda, Y.H. Ohtsuki. Theory of diffracted channeling radiation // Physical Reviw B. 2001, v.63, p.174112-1174112-7.

23. O.V. Bogdanov, Yu.L. Pivovarov. K.B. Korotchenko, diffracted X-ray radiation at (111) channeling of electrons in $\mathrm{Si}$ : ifluence of zone structure of energy levels // Pis'ma v Zh. Exp. Teor. Fiz. 2007, v.85, N11, p. 684-688 (in Russian).
24. H. Uberal. High-Energy Inerference Effect of Bremsstrahlung and Pair Production in Crystals // Phys. Rev. 1956, v.103, N4, p.1055-1067.

25. G. Lutz, U. Timm. Coherent Bremsstrahlung and Pair Production in the Diamond Crystal in Graphical Bepresentation between 1 and $40 \mathrm{GeV}$ // Z. Naturforschung. 1966, v.21a, p.1976-1987.

26. Giordano Diambrini Palazzi. High-Energy Bremsstrahlung and Electron Pair Production in Thin Crystal // Reviews of Modern Physics. 1968, v.40, N3, p.611-631.

27. V.L. Morokhovskii. All types of coherent radiations, which arise in the collision of relativistic electrons with crystal // Problems of Atomic Science and Technology. Series: "Nuclear Physics Investigations". 2015, N3(97), p.109-122.

28. V.L. Morokhovskii. Channeling of relativistic electrons and positrons // Problems of Atomic Science and Technology. Series: "Nuclear Physics Investigations". 2009, N5(63), p.122-129.

29. V.L. Morokhovskii. Coherent polarization radiation of relativistic electrons in crystals // Problems of Atomic Science and Technology. Series: "Nuclear Physics Investigations". 2014, N5(93), p.124-135.

30. V. L. Morokhovskii. Dynamics of relativistic electrons (positrons) in crystal: Preprint KIPT 8240, Kharkov, 1982, 22 p. (in Russian).

\title{
ЗАМЕТКИ О КОГЕРЕНТНОМ ПОЛЯРИЗАЦИОННОМ РЕНТГЕНОВСКОМ ИЗЛУЧЕНИИ РЕЛЯТИВИСТСКИХ ЭЛЕКТРОНОВ В КРИСТАЛЛЕ
}

\author{
В. Л. Мороховский
}

Рассмотрены ограничения применимости так называемой кинематической теории когерентного поляризационного рентгеновского излучения релятивистских электронов в монокристаллической мишени.

\section{НОТАТКИ ПРО КОГЕРЕНТНЕ ПОЛЯРИЗАЦІЙНЕ РЕНТГЕНІВСЬКЕ ВИПРОМІНЮВАННЯ РЕЛЯТИВІСТСЬКИХ ЕЛЕКТРОНІВ У КРИСТАЛІ}

\section{В. Л. Мороховсъкий}

Розглянуто обмеження застосування так званої кінематичної теорії когерентного поляризаційного рентгенівського випромінювання релятивістських електронів у монокристалічній мішені. 